\title{
Digital carbonate rock physics
}

\author{
Erik H. Saenger ${ }^{1,2}$, Stephanie Vialle ${ }^{3}$, Maxim Lebedev ${ }^{3}$, David Uribe ${ }^{4,5}$, Maria Osorno ${ }^{4}$, Mandy Duda ${ }^{1}$, and \\ Holger Steeb ${ }^{4,5}$ \\ ${ }^{1}$ International Geothermal Centre, 44801 Bochum, Germany \\ ${ }^{2}$ Institute for Geology, Mineralogy and Geophysics, Ruhr-Universität Bochum, 44801 Bochum, Germany \\ ${ }^{3}$ Department of Exploration Geophysics, Curtin University, Perth, Australia \\ ${ }^{4}$ Institute for Mechanics, University of Stuttgart, 70569 Stuttgart, Germany \\ ${ }^{5}$ Stuttgart Research Centre for Simulation Technology (SRC SimTech), 70569 Stuttgart, Germany
}

Correspondence to: Erik H. Saenger (erik.saenger@rub.de)

Received: 1 March 2016 - Published in Solid Earth Discuss.: 9 March 2016

Revised: 7 July 2016 - Accepted: 13 July 2016 - Published: 4 August 2016

\begin{abstract}
Modern estimation of rock properties combines imaging with advanced numerical simulations, an approach known as digital rock physics (DRP). In this paper we suggest a specific segmentation procedure of $\mathrm{X}$-ray microcomputed tomography data with two different resolutions in the $\mu \mathrm{m}$ range for two sets of carbonate rock samples. These carbonates were already characterized in detail in a previous laboratory study which we complement with nanoindentation experiments (for local elastic properties). In a first step a non-local mean filter is applied to the raw image data. We then apply different thresholds to identify pores and solid phases. Because of a non-neglectable amount of unresolved microporosity (micritic phase) we also define intermediate threshold values for distinct phases. Based on this segmentation we determine porosity-dependent values for effective $P$ - and $S$-wave velocities as well as for the intrinsic permeability. For effective velocities we confirm an observed twophase trend reported in another study using a different carbonate data set. As an upscaling approach we use this twophase trend as an effective medium approach to estimate the porosity-dependent elastic properties of the micritic phase for the low-resolution images. The porosity measured in the laboratory is then used to predict the effective rock properties from the observed trends for a comparison with experimental data. The two-phase trend can be regarded as an upper bound for elastic properties; the use of the two-phase trend for low-resolution images led to a good estimate for a lower bound of effective elastic properties. Anisotropy is observed for some of the considered subvolumes, but seems to be insignificant for the analysed rocks at the DRP scale. Because
\end{abstract}

of the complexity of carbonates we suggest using DRP as a complementary tool for rock characterization in addition to classical experimental methods.

\section{Introduction}

Three-dimensional information on rock microstructures is important for a better understanding of physical phenomena and for rock characterization on the microscale. Various destructive and non-destructive methods for obtaining a 3-D image of the rock microstructure exist (Arns et al., 2002; Saenger et al., 2004; Madonna et al., 2013; Cnudde and Boone, 2013; Wang et al., 2013, and references therein). The most common non-destructive 3-D imaging technique for rock samples is X-ray computed tomography (XRCT). A common problem, however, is a clear trade-off between sample size and resolution. For each material a specific and large enough sample size is required to ensure that the selected volume is representative of the physical property to be computed (e.g. Hill, 1963; Costanza-Robinson et al., 2011; Andrä et al., 2013a). It can, however, be at the expense of a loss of pore feature resolution. In the last decade, the Xray micro-computed tomography (micro-XRCT) method became widely available and many modern studies have made use of it to obtain 3-D rock images (e.g. Fusseis et al., 2014). The resolution of micro-XRCT of up to $(0.6 \mu \mathrm{m})^{3}$ (voxel size) is high enough to image the spatial distribution of grains, pores, and pore fluids for a wide variety of rocks (e.g. Blunt et al., 2013; Madonna et al., 2013). 
Rock images from 3-D XRCT can be used for predicting properties such as porosity, permeability, pore size distribution, effective elastic moduli, or electrical conductivity (e.g. Andrä et al., 2013b). For example, Darcy permeability can be predicted by numerically simulating single-phase fluid flow through 3-D rock pore structure models, with the numerical results being in reasonable agreement with laboratory measurements (e.g. Osorno et al., 2015; Saenger et al., 2016). In this case, the resolution of the micro-XRCT technique is sufficient, because fluid pathways predominantly follow larger pores. However, if the pore size is much smaller than $1 \mu \mathrm{m}$ the agreement might be less satisfactory due to resolution limitations. On the other hand, mechanical properties, such as the effective elastic moduli, strongly depend on the microstructural details of the rock, which may stay unresolved by the micro-XRCT technique (e.g. for Bentheim sandstone considered in Saenger et al., 2016). The inability to fully characterize the microstructural details of a rock sample can lead to a disagreement between numerical estimates of mechanical properties based on micro-XRCT images and laboratory data (Andrä et al., 2013b).

An example of such a disagreement between laboratory and digital rock physics (DRP) estimates is described in Andrä et al. (2013a, b). In these benchmark papers a comparison between different numerical methods is presented. All DRP estimates of the effective elastic bulk modulus use the same segmented data set. Regardless of the numerical approach, all computational predictions overestimate the bulk modulus measured in the laboratory. This conclusion is mostly based on Berea sandstone although carbonates are considered in this study. However, Jouini et al. (2015) also reports about an overestimation of effective elastic properties of carbonates by DRP. Therefore we conclude here that the digital rock images themselves and/or the computational workflow have to be improved to provide better estimates of effective properties of rocks. In this paper we consider a carbonate data set in detail and suggest techniques to achieve a better agreement between numerical predictions and laboratory measurements. Our study is complementary to the DRP carbonate studies performed in Derzhi et al. (2010), Lopez et al. (2012), Ringstad et al. (2013), Andrä et al. (2013a, b), and Jouini et al. (2015). In contrast to these studies our digital rock physics study is complemented with a very detailed experimental characterization (Sect. 2). Our suggested segmentation technique (Sect. 3) is used to estimate effective mechanical and transport properties (Sect. 4). Among others, we observe a two-phase trend for monomineralic (calcite) carbonates which can be regarded as an upper bound for velocities at all scales (see discussion in Sect. 5) due to the observed self-similarity of those rocks (Jouini et al., 2015).

\section{Rock samples and laboratory characterization}

\subsection{Carbonate samples}

We studied samples of two carbonates from the Upper Cretaceous carbonate system of the Gargano-Murge region (southern Italy). Carb-A is a limestone from the PaleoceneEocene Peschici Formation, and Carb-B is a micritic mudstone from the Late Cretaceous Monte Acuto Formation (Martinis and Pavan, 1967; Cremonini et al., 1971). Both carbonates are composed of nearly $100 \%$ calcite (Scotellaro et al., 2014).

Both samples have been characterized in the laboratory in detail in Vialle et al. (2013), e.g. SEM images showing the microstructure of these two samples. We only give a short summary here. Both carbonate samples display a matrix of micrite (abbreviation for microcrystalline calcite) with a typical grain size of $1-4 \mu \mathrm{m}$ (Moshier, 1989), but the texture of these micrites is different between the two samples. Following the classification of micrite microtexture proposed by Lambert et al. (2006) and Deville de Periere et al. (2011), the micrite in sample Carb-A is mainly a "tight micrite", spatially varying from anhedral compact to fused, with grains typically $1-2 \mu \mathrm{m}$ in diameter; micrite in sample Carb-B is a "porous micrite", varying from rounded to subrounded, with anhedral to subhedral, rounded grains, typically $2-4 \mu \mathrm{m}$ in diameter. Besides a micrite matrix, sample Carb-A exhibits vuggy-like pores either rounded, up to about $60 \mu \mathrm{m}$, or more elongated, up to $300 \mu \mathrm{m}$ in length. Sample Carb-B exhibits a calcite spar cement of grains typically tens to hundreds of micrometres in diameter, as well as rounded vugs up to about $100-200 \mu \mathrm{m}$.

He-grain density, bulk density, and resulting porosity, as well as air permeability, were previously measured at room pressure and temperature on core plugs $0.025 \mathrm{~m}$ in length and diameter (Vialle et al., 2013). The associated errors did not exceed $0.5,1$, and $2 \%$, respectively. $P$ - and $S$-wave velocities, at 1 and $0.7 \mathrm{MHz}$ frequency, respectively, were acquired on dry samples under increasing (up to $30 \mathrm{MPa}$ ) and decreasing hydrostatic stress. Velocities were measured by using a pulse-transmission technique. The errors in $V_{P}$ and $V_{S}$ were about $1 \%$. The results are listed in Tables 1 and 2 . The obtained grain densities $\left(2690 \pm 10\right.$ and $2700 \pm 10 \mathrm{~kg} \mathrm{~m}^{-3}$ for samples Carb-A and Carb-B, respectively) are in agreement with a mineralogy of pure calcite (Mavko et al., 2009).

\subsection{Nanoindentation}

Nanoindentation tests were performed to obtain stiffness (Young's modulus) of the carbonates at the micrometre scale. These tests were performed on room-dried samples consisting of two small irregular pieces, about $5 \mathrm{~mm}$ thick and with a surface of a few $\mathrm{cm}^{2}$, taken from the cuttings of the $0.025 \mathrm{~m}$ core plugs of Carb-A and Carb-B. Prior to testing, the surface of each sample was polished with carbide paper (grit 120). 
Table 1. Helium bulk and grain density (in $\mathrm{g} \mathrm{cm}^{-3}$ ), helium porosity (in PU), and air permeability (in $\mathrm{mD}$ ) measured at benchtop conditions for Carb-A and Carb-B. Permeability of plug Carb-A is below the sensitivity level of the apparatus used (0.1 mD). Values are from Vialle et al. (2013).

\begin{tabular}{lcccr}
\hline Sample ID & Porosity & Bulk density & Grain density & Permeability \\
\hline Carb-A & $0.167 \pm 0.002$ & $2.24 \pm 0.01$ & $2.69 \pm 0.01$ & $<0.1$ \\
Carb-B & $0.294 \pm 0.003$ & $1.90 \pm 0.01$ & $2.70 \pm 0.01$ & $60 \pm 5$ \\
\hline
\end{tabular}

Table 2. Pressure dependence of the elastic wave velocities for the two studied samples. Pressure is in megapascal, and $P$ - and $S$-wave velocity are in kilometres per second. Values are from Vialle et al. (2013).

\begin{tabular}{lcc|cc}
\hline \multirow{2}{*}{ Pressure } & \multicolumn{2}{c|}{ Carb-A } & \multicolumn{2}{c}{ Carb-B } \\
\cline { 2 - 5 } & $V_{P}$ & $V_{S}$ & $V_{P}$ & $V_{S}$ \\
\hline 0 & 5.007 & 2.835 & 2.769 & 1.754 \\
2.5 & 5.045 & 2.838 & 3.295 & 2.032 \\
7.5 & 5.091 & 2.835 & 3.477 & 2.191 \\
10 & 5.100 & 2.834 & 3.504 & 2.226 \\
20 & 5.116 & 2.822 & 3.551 & 2.295 \\
30 & 5.113 & 2.821 & 3.553 & 2.290 \\
25 & 5.114 & 2.821 & 3.548 & 2.284 \\
15 & 5.085 & 2.822 & 3.527 & 2.268 \\
5 & 5.048 & 2.840 & 3.384 & 2.195 \\
0 & 5.033 & 2.842 & 2.792 & 1.785 \\
\hline
\end{tabular}

Roughness (Sq) of the surface measured by DS 95 AFM system (Semilab) on $10 \mu \mathrm{m} \times 10 \mu \mathrm{m}$ areas was $1.4 \mu \mathrm{m}$ (rms values) for Carb-A and not measured for Carb-B. The IBIS nanoindentation system (Model B, Fisher-Cripps Laboratories Pty.Ltd.) is equipped with a Berkovich-type diamond indenter (Lebedev et al., 2014) and was used in static mode: the tests consist of continuously recording the load, $P$, and the displacement, $h$, of the indenter as it pushes into and withdraws from the surface of the sample. A constant maximum loading force of $10 \mathrm{mN}$ and an initial contact force of $0.15 \mathrm{mN}$ were used. In total, $961(31 \times 31)$ measurements were performed on a $300 \times 300 \mu \mathrm{m}$ surface with a spacing of $10 \mu \mathrm{m}$ between measurement points.

Typically, the extraction of the mechanical properties is achieved by using the $P$ - $h$ curves and by applying a continuum-scale mechanical model to obtain the indentation modulus M (Fischer-Cripps, 2004; Lebedev et al., 2014):

$M \stackrel{\text { def }}{=} \frac{\sqrt{\pi}}{2} \frac{S}{\sqrt{A_{\mathrm{c}}}}$

where $S$ the is unloading indentation stiffness $S=(\mathrm{d} P / \mathrm{d} h)$ and $A_{\mathrm{c}}$ the contact area, extrapolated from the maximum penetration depth $h_{\max }$ and using the relation $A_{\mathrm{c}}=24.5 h_{\max }^{2}$ according to the geometry of Berkovitch-type indenters (Fischer-Cripps, 2004).
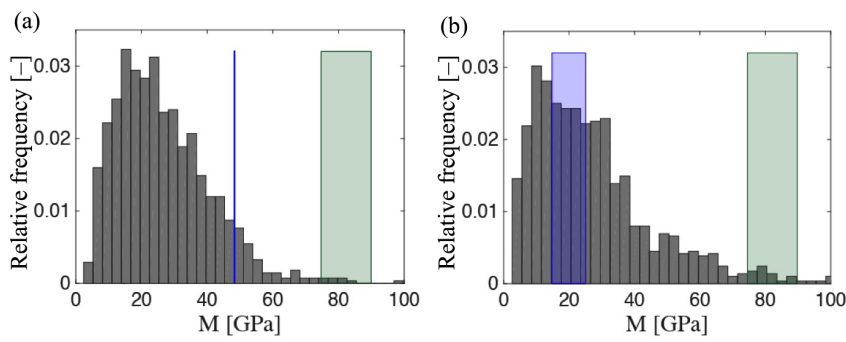

Figure 1. Nanoindentation results for Carb-A (left-hand side) and Carb-B (right-hand side). In blue we illustrate the corresponding moduli range from ultrasonic experiments on dry samples from 0 to $30 \mathrm{MPa}$ confining pressure, and in green we illustrate the moduli range given by the solid anisotropic calcite crystal. Overall we observe that the medium effective indentation module $\mathrm{M}$ is slightly stiffer for Carb-A (26 GPa vs. $25 \mathrm{GPa}$ ).

Data were further corrected considering deviation of the indenter tip from ideal geometry, initial penetration into the rock below a load threshold, and compliance of the loading column, leading to a nominal uncertainty of indentation moduli of $<2 \mathrm{GPa}$.

Young's moduli, E, can be calculated from the indentation moduli (Fischer-Cripps, 2004) according to

$\frac{1}{M}=\frac{1-v^{2}}{E}+\frac{1-v_{i}^{2}}{E_{i}}$.

Indenter properties are $E_{i}=1220 \mathrm{GPa}$ and $E_{i}=0.06$, according to Klein and Cardinale (1992) and FischerCripps (2004) for diamond material. Each performed measurement covers a projected surface of about $40 \mu \mathrm{m}^{2}$ on average (which corresponds to an equilateral triangle with a side of $6 \mu \mathrm{m}$ ) and contains both pores and solid grains. A Poisson's ratio has also to be assumed for each nanoindentation measurement: even though we can compute it from the laboratory ultrasonic $P$ - and $S$-wave measurements at the core scale, there is no reason to assume that this value is constant for each individual measurement. This local Poisson's ratio cannot be measured experimentally and we have taken here a constant value of 0.3 . Figure 1 displays the distribution of the indentation moduli for both Carb-A (left) and Carb-B (right). 


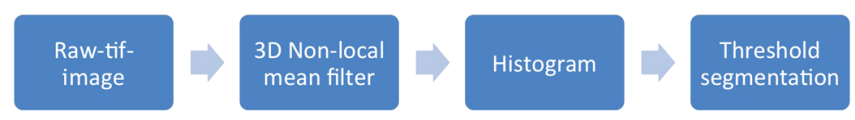

Figure 2. Simplified segmentation workflow as applied in this study.

\section{The CT data sets}

\subsection{Procedure to get $\mathrm{CT}$ images}

Two samples were prepared for imaging with micro-XRCT from the cuttings, one from Carb-A and one from Carb-B. A cylindrical-shaped sample of $1.5 \mathrm{~cm}$ in height and $2 \mathrm{~mm}$ in diameter was achieved by gently grinding the cuttings, first on the side on a rock saw blade, then by hand using sand paper (grit 120). This procedure allows us to obtain very thin cylinders while minimizing mechanical damage that classical drilling would produce. These cylindrical samples were then glued with Crystalbond509 (SPI suppliers) on a $2 \mathrm{~mm}$ diameter flat-head metal pin, which was itself inserted in the core holder of the micro-tomograph. The 3-D X-ray Microscope Versa XRM 500 (Zeiss - XRadia) was used with a $\mathrm{X}$-ray energy of $60 \mathrm{keV}$. Two different settings of source-tosample and detector-to-sample distance were used to achieve two nominal voxel sizes of $(3.4 \mu \mathrm{m})^{3}$ and $(0.6 \mu \mathrm{m})^{3}$ for Carb$\mathrm{A}$, and of $(3.4 \mu \mathrm{m})^{3}$ and $(1.1 \mu \mathrm{m})^{3}$ for Carb-B, referred to as "low resolution" and "high resolution", respectively, for each for the two samples. X-ray microscope and image acquisition settings are summarized in Table 3 for each of the two samples.

The number of radiographic projections acquired during sample imaging with low and high resolutions were 3001 and 5001, respectively. The total scanning time for one sample was about $8 \mathrm{~h}$. Initial cone-beam 3-D image reconstruction was performed using the internal software XM Reconstructor (XRadia). To remove geometrical artefacts during reconstruction, a secondary reference was acquired for sample images with maximum resolution.

\subsection{Segmentation procedure}

In addition to solid grains and pore space different micritic phases are visible in the raw images of the scans entailing an advanced segmentation procedure. For our segmentation (Fig. 2) we select a region of interest (ROI) from the raw data of the two types of carbonates with two different resolutions (Table 3). The ROI is subdivided in eight partly overlapping subvolumes, each of a size of $400^{3}$ voxels (Figs. 3 and 4). For the low-resolution images, it thus gives eight cubes with a side of $1.37 \mathrm{~mm}$ for both samples, and for the low-resolution images, eight cubes with a side of $0.25 \mathrm{~mm}$ for Carb-A and of $0.46 \mathrm{~mm}$ for Carb-B.

Our segmentation workflow is applied to the full ROI including all subvolumes. Image enhancement and segmenta-

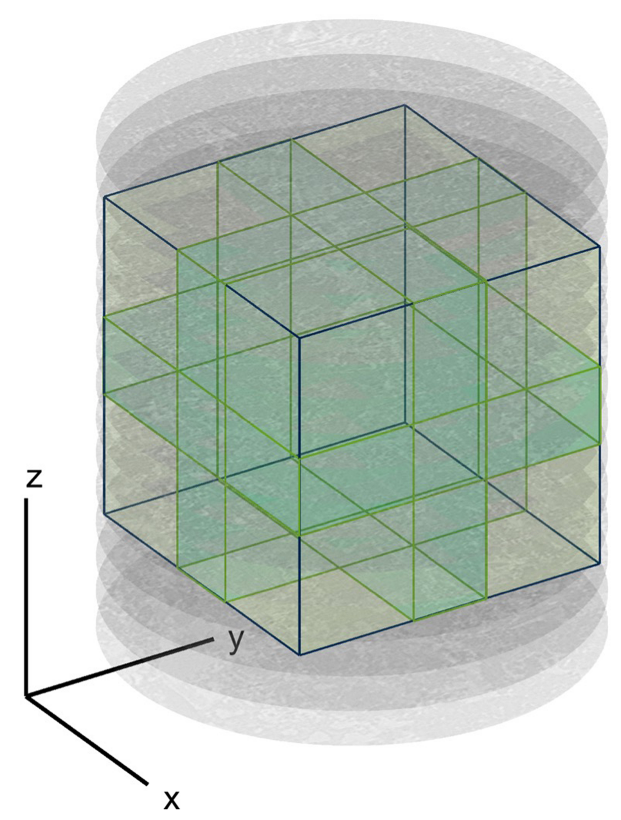

Figure 3. Sketch to illustrate the segmentation geometry (here for Carb-B; high resolution). The full cube is subdivided into eight partly intersecting subvolumes with a size of $400^{3}$ grid points. Those subvolumes are used in the numerical simulations to estimate effective material properties.

tion steps were carried out using the software package Avizo Fire 9 (FEI Visualization Sciences Group). Before actual segmentation the image noise and scan artefacts are reduced while preserving interfaces using a 3-D non-local mean image filter. In our experience the standard values of this filter are appropriate (search windows $=21$, local neighborhood $=5$, similarity value $=0.6$ ). Note that every step of image enhancement changes the original data set, affecting subsequent steps required for data analysis.

The image-enhanced data sets were segmented into classes using global thresholds for the covered range of grey values. The global threshold is valid for all the eight partly overlapping subvolumes mentioned above. Considering that the samples represent quasi-monomineralic calcitic rocks, we identified the following classes illustrated in Figs. 5 and 6:

- high-confidence pores (illustrated with dark blue colour),

- high-confidence mineral (illustrated with dark red colour),

- and five intermediate classes.

Note that a non-negligible part of the pore space is below the resolution limit of the $\mu$-CT scans (cf. results of mercury intrusion showing pores and pore throats as low as $0.06 \mu \mathrm{m}$, Fig. 6 of Vialle et al. (2013)). We found that five intermediate classes are sufficient to describe the calcitic rocks used in this study, but the number of classes between the high-confidence 
Table 3. CT scanner parameters used for image acquisition of the two carbonate samples. Sample abbreviations are explained in Table 1.

\begin{tabular}{lll|ll}
\hline & \multicolumn{2}{c}{ Carb-A } & \multicolumn{2}{c}{ Carb-B } \\
\cline { 2 - 5 } Pressure & Low resolution & High resolution & Low resolution & High resolution \\
\hline Voxel size & $(3.4348 \mu \mathrm{m})^{3}$ & $(0.6245 \mu \mathrm{m})^{3}$ & $(3.4352 \mu \mathrm{m})^{3}$ & $(1.1450 \mu \mathrm{m})^{3}$ \\
Image size & $1012 \times 1012$ & $1012 \times 1012$ & $1013 \times 1013$ & $1013 \times 1013$ \\
Acceleration voltage & $60 \mathrm{kV}$ & $60 \mathrm{kV}$ & $60 \mathrm{kV}$ & $60 \mathrm{kV}$ \\
Current & $80 \mu \mathrm{A}$ & $80 \mu \mathrm{A}$ & $83 \mu \mathrm{A}$ & $83 \mu \mathrm{A}$ \\
Source-to-sample distance & $50.00 \mathrm{~mm}$ & $11.10 \mathrm{~mm}$ & $12.00 \mathrm{~mm}$ & $12.00 \mathrm{~mm}$ \\
Detector-to-sample distance & $50.00 \mathrm{~mm}$ & $120.00 \mathrm{~mm}$ & $12.00 \mathrm{~mm}$ & $60.00 \mathrm{~mm}$ \\
Exposure time & $20 \mathrm{~s}$ & $20 \mathrm{~s}$ & $1 \mathrm{~s}$ & $6 \mathrm{~s}$ \\
Optical magnification & $4 \times$ & $4 \times$ & $4 \times$ & $4 \times$ \\
\hline
\end{tabular}
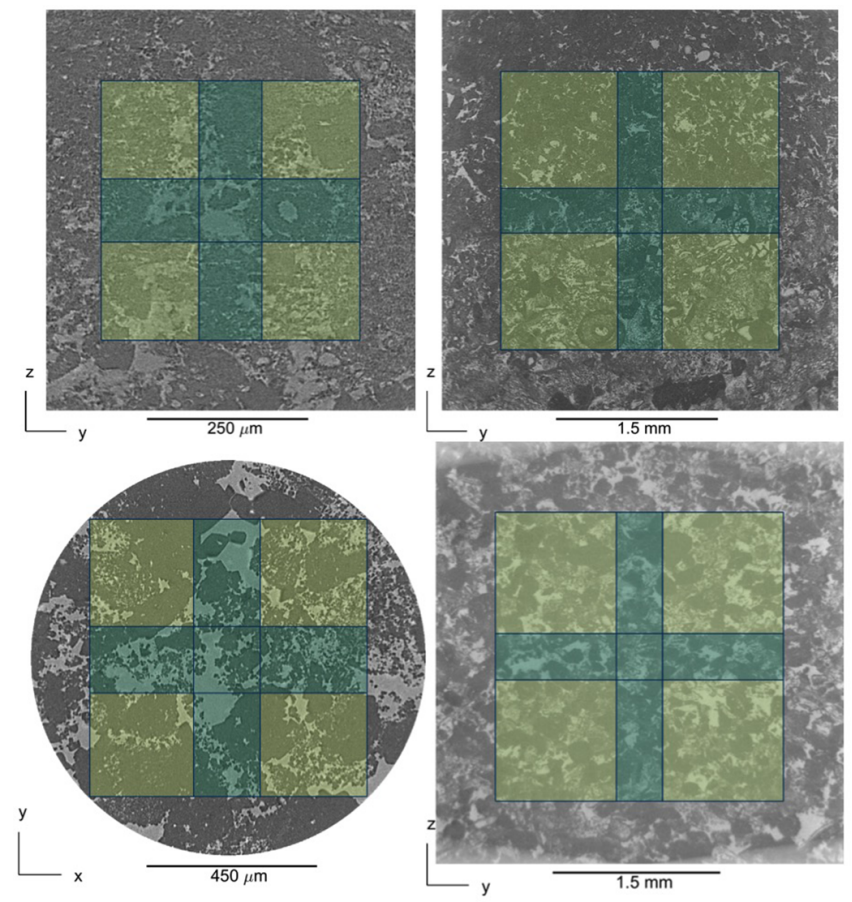

Figure 4. Slices of the raw tiff images of the scanned samples. The dark green areas mark the overlapping zones of the considered subvolumes. Top row: Carb-A with high (left) and low resolution (right). Bottom row: Carb-B with high (left) and low resolution (right).

mineral class and the high-confidence pore class can be increased or decreased for other materials. In contrast to Lopez et al. (2012) or Ringstad et al. (2013), we do not think that the density can be approximated directly by the grey value of those micritic phases. From our point of view the relationship between grey value and density can be highly non-linear and becomes even more complicated in the case of multimineral rocks. However, we make use of the accepted assumption that lighter grey values in the histogram correspond to lower porosities.

\section{Numerical results}

In order to numerically calculate the effective intrinsic permeability $k$ of the digitized rock sample, we calculate the fluxes under creeping flow condition based on a parallelized Stokes solver. The parallelized finite-difference-based Stokes solver is suitable for the calculation of effective hydraulic parameters for low and high porous materials (cf. Osorno et al., 2015). Using volume averaging technique, we coarse-grain the local velocity field $\boldsymbol{u}(\boldsymbol{x})$, obtaining the global velocity component $\boldsymbol{u}_{m}$ in flow direction.

The intrinsic permeability $k$ is calculated with Darcy's law:

$k=\frac{\mu \boldsymbol{u}_{m}}{\Delta p}$.

The pressure gradient $\Delta p$ is imposed with pressure boundary conditions in the numerical simulations. The dynamic viscosity of the pore fluid is $\mu$. In our numerical simulations $\Delta p$ is $-5.8 \times 10^{-4} \mathrm{~Pa} \mathrm{~m}^{-1}$ and $\mu$ is $1.2 \mathrm{Pas}$.

To obtain effective $P$ - and $S$-wave velocities of the digitized rock samples we use a technique described in detail in Saenger et al. (2011) and references therein. The basic idea of this approach is to study speeds of elastic waves through heterogeneous materials in the long wavelength limit (pore size « wavelength) using the rotated staggered grid (RSG) finite difference algorithm (Saenger et al., 2000) for the simulation of elastic wave velocities (cf. Andrä et al., 2013a, b).

\subsection{High resolution}

\subsubsection{Permeability}

Permeability calculations were realized for subvolumes of the Carb-A and Carb-B samples. However, the domain size of the Carb-A $(0.43 \mathrm{~mm})$ and Carb-B $(0.78 \mathrm{~mm})$ highresolution samples is smaller than the low-resolution ones ( $2.4 \mathrm{~mm}$ for Carb-A and Carb-B), i.e. less representative of the material; therefore we numerically investigate only the extreme porosity configurations. To select the domains for permeability calculation we adapt the porosity configurations 

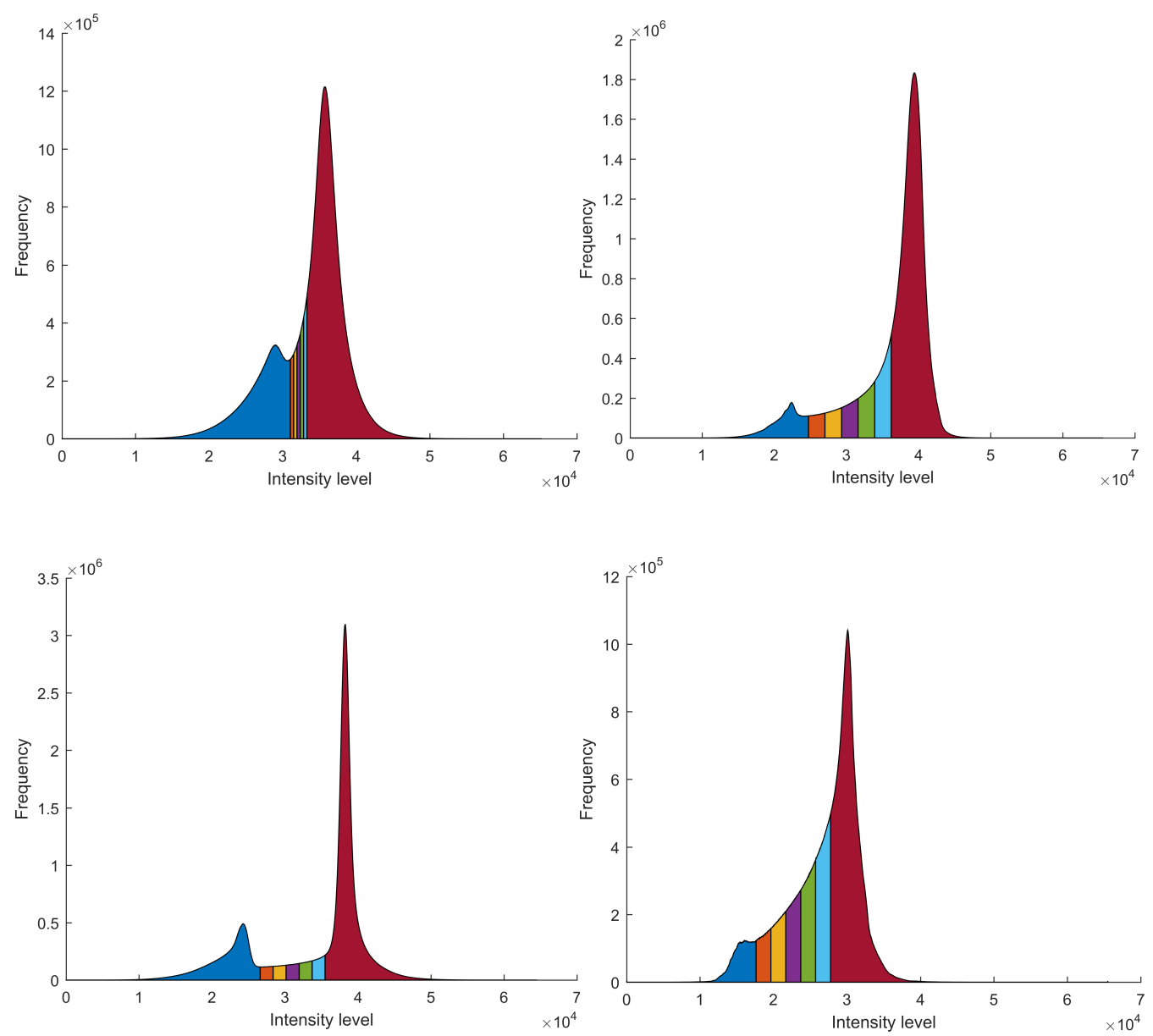

Figure 5. Colour-coded histograms of the scanned samples. Top row: Carb-A with high (left) and low resolution (right). Bottom row: Carb-B with high (left) and low resolution (right). Between the high-confidence pore phase (marked blue) and the high-confidence mineral phase (marked red) we define five intermediate classes to characterize the micritic phases within carbonate rock.

(range defined by high-confidence pores to high-confidence grains; see also discussion in Sect. 5.2) showing the minimum and maximum deviation in porosity with respect to the experimental investigation. To analyse the homogeneity of the sample this step was performed for all eight subvolumes. For the high-resolution subdomains (Carb-A and Carb-B) we perform Stokes flow simulations only in one direction $(z$ direction, cf. Fig. 3).

The left-hand side of Fig. 7 displays the intrinsic permeability calculated for the Carb-A high resolution. The porosity range of the subvolumes is higher than the experimentally determined porosity. In addition, the numerically calculated permeability values are significantly lower than the values obtained for the low-resolution samples. The right-hand side of Fig. 7 shows the results of the intrinsic permeability calculated for the selected high-resolution samples of the CarbB. It can be observed that the high-resolution sample shows a much lower variation between the extreme values of the porosity range.
From the results of the high-resolution samples, Carb-A and Carb-B, it could be observed that the variation in pore channel arrangement is significant and the permeability in the different subvolumes of the same material does not necessarily increase with the porosity increment.

\subsubsection{Elasticity}

Several micritic phases have been identified in the raw images of the carbonate rock (i.e. the phases between highconfidence pores and high-confidence minerals; compare with Sect. 3.2). The porosity of these regions cannot be determined exactly, as some pores are below the resolution of the scans: typically, micrites exhibit pore sizes with a maximum diameter of $1 \mu \mathrm{m}$ (Moshier, 1989; Cantrell and Hagerty, 1999), and pore sizes as low as $0.06 \mu \mathrm{m}$ have been measured by mercury intrusion capillary pressure tests (MICP) for the samples under investigation (Vialle et al., 2013). To account for the unresolved pore space we perform a number of two-phase wave propagation simulations to estimate effec- 

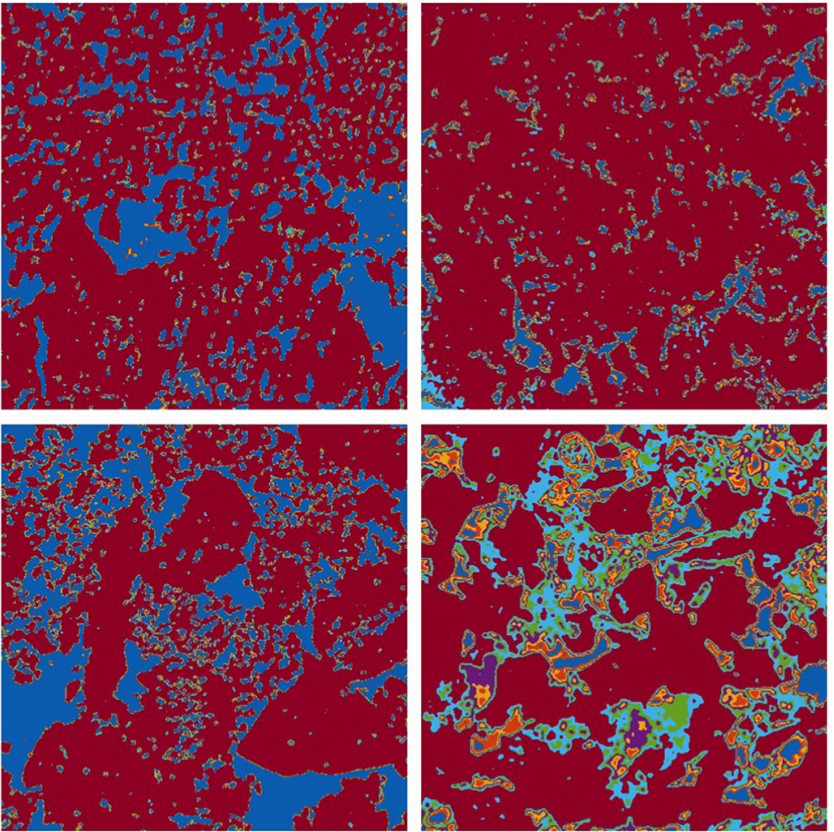

Figure 6. Slices of the segmented images used for the numerical simulations to determine permeability and velocities. Top row: Carb-A with high (left) and low resolution (right). Bottom row: Carb-B with high (left) and low resolution (right).

tive elastic properties (Saenger et al., 2004, 2011, 2016). For these time-of-flight simulations we assign vacuum properties to the pore phase, while the rest of the digital image including the micritic phase will be assumed to be solid with the mineral properties of calcite (e.g. Andrä et al., 2013b). For the second simulation, we assign vacuum properties to the pores and the first micritic phase, while the rest will be assumed to be solid. We continue this way for all micritic phases, so that the last simulation assigns the mineral properties of calcite only to the high-confidence mineral phase. By this technique we obtain a porosity-velocity trend (Fig. 8) for a random selection of high-resolution subvolumes for Carb-A and CarbB:

$$
\begin{aligned}
& V_{P}=6259.1 \mathrm{~m} \mathrm{~s}^{-1}-\varphi \cdot 9640 \mathrm{~ms}^{-1}+3381 \mathrm{~m} \mathrm{~s}^{-1} \cdot \varphi^{2} \\
& V_{S}=3237.2 \mathrm{~ms}^{-1}-\varphi \cdot 3237.2 \mathrm{~ms}^{-1} .
\end{aligned}
$$

This porosity-velocity trend is exactly the same as has been observed for a carbonate data set from a different location used in Saenger et al. (2014). In their paper this trend has been observed for three different resolutions $(65 \mathrm{~nm}, 1$ and $4 \mu \mathrm{m})$. Please note that due to computational restrictions we are only able to simulate a random selection of subsamples; however, as shown in Fig. 8, all our calculated velocities follow the trends according to Eqs. (4) and (5).

\subsection{Low resolution}

\subsubsection{Permeability}

Similar to the procedure of the numerical simulation for elasticity (Sect. 4.1.2), we vary the sample porosity. This way we get six different porosities for each subvolume depending on the threshold variation. To reduce computational times for the Stokes flow simulation we eliminate the disconnected pores. Some subvolume solid-pore configurations with lower porosities do not present connected pores, and we assume the effective permeability $k=0$.

Figure 9 presents the permeability values for Carb-A (lefthand side) and Carb-B (right-hand side) samples as a function of porosity. For the permeability calculations for these samples we perform the Stokes flow simulation in a $\mathrm{z}$ direction only (compare Fig. 3).

Additionally we performed the Stokes flow simulations in three directions $(X, Y$, and $Z$ ) for Carb-A sample (see Fig. 11). From the simulation results it can be seen that the Carb-A sample permeability (Fig. 11, left-hand side) is anisotropic with a variation between directions of up to $80 \%$. In some subvolumes permeability value varies by up to 2 orders of magnitude.

From the CT data of the low-resolution CarbA, the largest domain that could be extracted is $2.4 \mathrm{~mm} \times 2.4 \mathrm{~mm} \times 2.4 \mathrm{~mm} \quad\left(689^{3}\right.$ voxels $)$. The permeability calculated for this domain is $13.0 \mathrm{D}$ for a porosity of $\varphi=0.173$.

\subsubsection{Elasticity}

For the low-resolution scans we repeat the two-phase simulations for Carb-A and Carb-B as described in Sect. 4.1.2. The results are displayed in Fig. 10. Interestingly, the two-phase trend given by Eqs. (4) and (5) is confirmed only clearly for $S$-wave velocities of Carb-A and Carb-B. For the case of $P$-wave velocities we observe a slightly different trend (cf. Eq. 4) that we illustrate with blue dashed/dotted lines in Fig. 10:

$V_{P}=6259.1 \mathrm{~ms}^{-1}-\varphi \cdot 7970 \mathrm{~ms}^{-1}+1700, \mathrm{~ms}^{-1} \cdot \varphi^{2}$.

Especially in the low-resolution case, we expect to have images with a large number of unresolved porosity, mainly due to micritic phases. Therefore we perform multiphase simulations and vary the porosity by assigning effective elastic properties to an interval of micritic phases (always starting with the class closest to the high-confidence pore phase). As an effective medium approach we use the trend given by the simulations using two single phases only (Eqs. 4 and 5), which is supported by two observations. First, this trend was already observed by Saenger et al. (2014) on different scales on a different carbonate data set. Second, there is an observed self-similarity for carbonates (Jouini et al., 2015). Therefore, despite the interval of micritic phases, we assign vacuum val- 

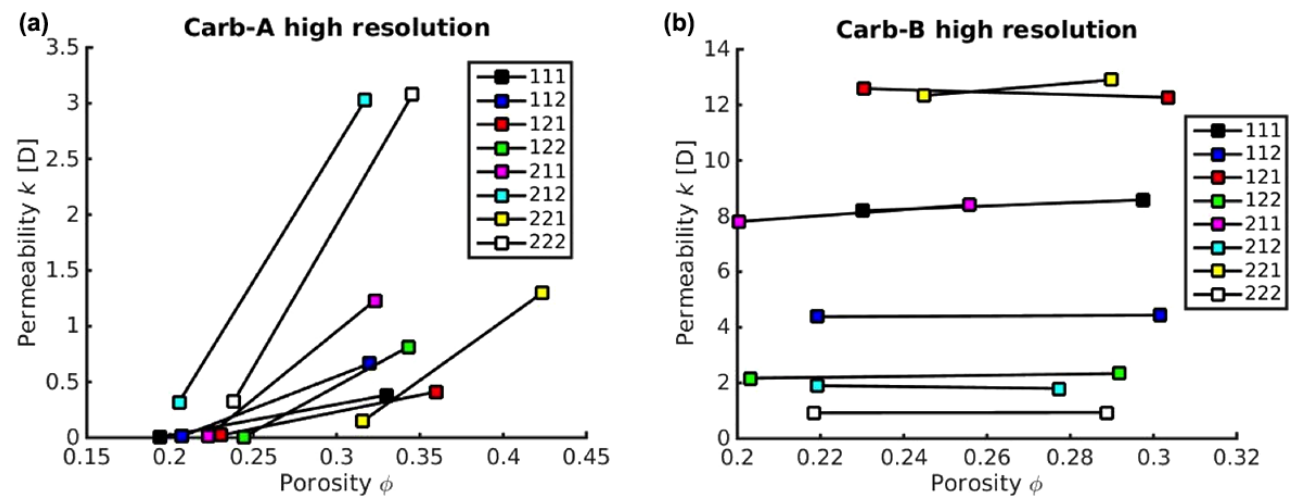

Figure 7. Intrinsic permeability simulated for the eight subvolumes. Results are given for the extreme porosities configuration of Carb-A (left-hand side) and Carb-B (right-hand side) high-resolution samples.
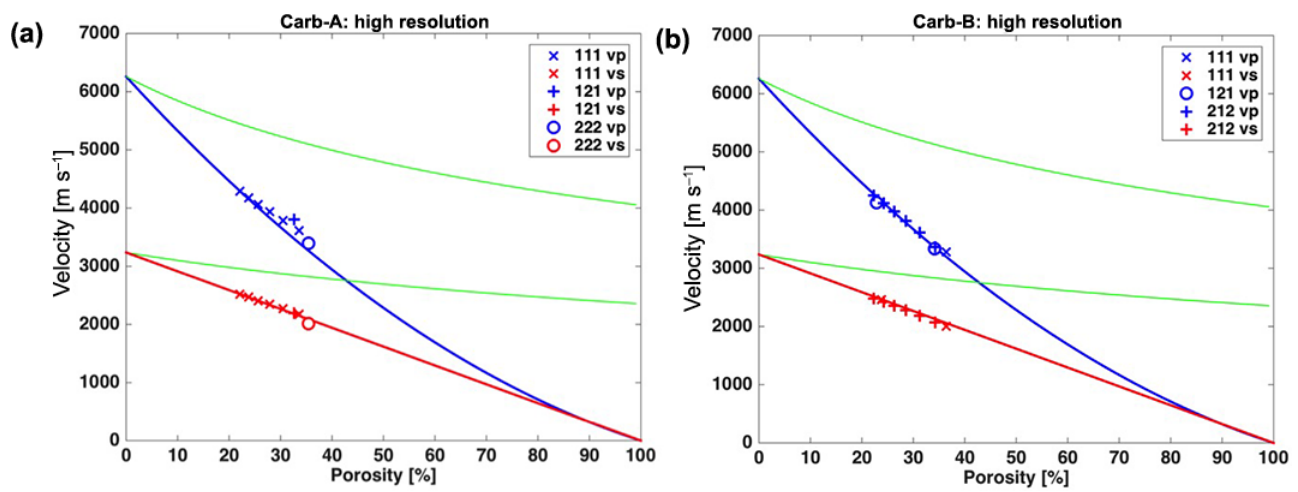

Figure 8. Effective $P$ - and $S$-wave velocities (red and blue dots, respectively) for simulations based on a two-phase segmentation. The results were obtained for a random selection of subvolumes of Carb-A (left-hand side) and Carb-B (right-hand side). The blue and red lines are the velocity trends given by Eqs. (4) and (5). The green lines are derived by using the upper Hashin-Shtrikman bounds. For details please refer to the text.

ues for the high-confidence pores and use the known elastic moduli of calcite (e.g. Andrä et al., 2013b) for the remaining phases. The results are displayed with green dots for Carb-B in Fig. 10. We repeat the procedure with different intervals of the micritic phases. There are three interesting observations: (1) the resulting effective velocities are always significantly below the observed two-phase trend, (2) the curves for different intervals will intersect each other, and (3) the experimental determined velocities for high confining pressures are between the multiphase results and the two-phase trend as described above.

\section{Discussion}

In this paper we compare results from laboratory investigations with numerical estimates based on digital images. Note that in laboratory experiments we use samples on the centimetre scale for the determination of permeability and ultrasonic velocities and compare it with DRP predictions based on images on the millimetre scale. Especially because of the known heterogeneity of carbonates there is always a risk that the selected scanned area is not representative compared to the full sample size used for laboratory characterizations. In general, a multiscale approach as suggested by Ringstad et al. (2013) should be used for upscaling the results to the plug scale. However, our studies on Carb-A and Carb-B suggest workflows which should be applied in practice for as many samples as possible for improving the statistical significance.

\subsection{Discussion of experimental characterization}

Even with the highest resolution currently available in microXRCT imaging there will be a significant amount of unresolved pore features which need to be treated in the DRP workflow (Saenger et al., 2016). On the grey-scale intensity level histograms of the low- and high-resolution images of the micro-CT scanning (Fig. 5), this is reflected in a continuum in the intensity levels between the phase identified as pores and the phase identified as calcite grains. In this paper we have dealt with these micritic phases by replacing, step by step and in a cumulative way, each of the micritic phases 

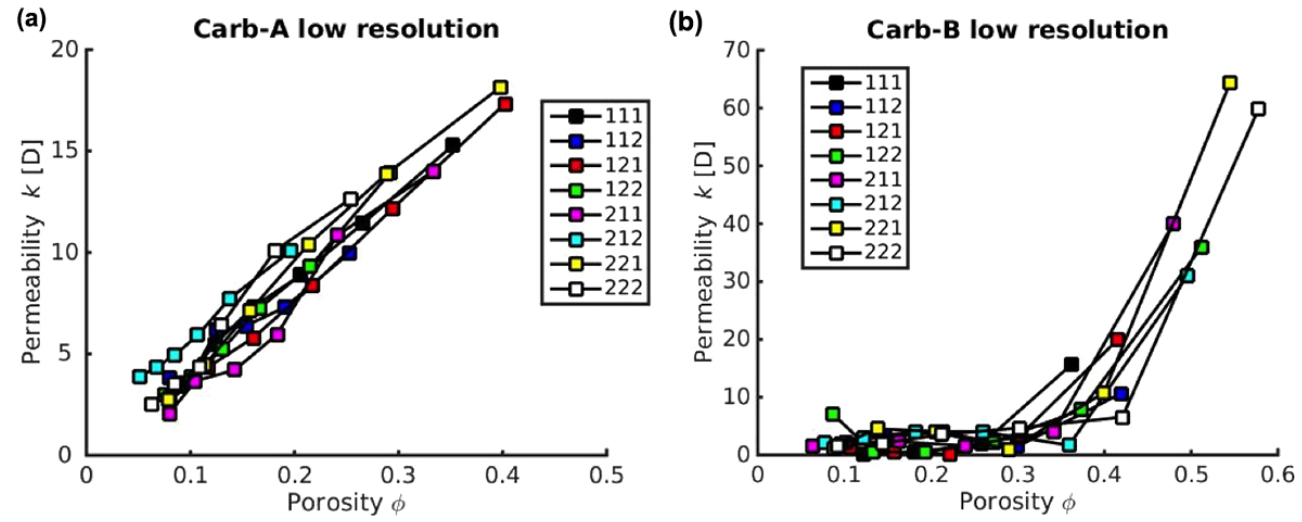

Figure 9. Simulated intrinsic permeability as a function of porosity. Results for the eight subvolumes of Carb-A (left-hand side) and Carb-B (right-hand side) low-resolution samples. Squares markers display each of the pore-solid configurations.
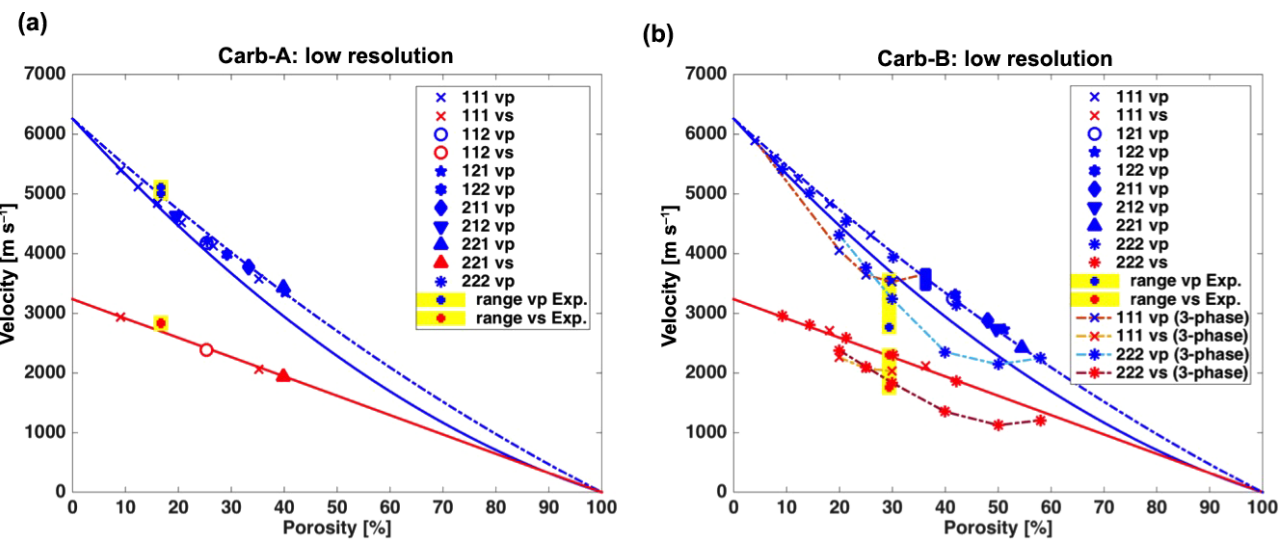

Figure 10. Effective $P$-wave and $S$-wave velocities (red and blue dots, respectively) for simulations based on a two-phase segmentation. The results were obtained for subvolumes of the low-resolution images of Carb-A (left-hand side) and of Carb-B (right-hand side). The blue and red lines are the velocity trends given by Eqs. (4) and (5); the blue dashed/dotted line is the trend given by Eq. (6). The experimental results (interval from 0 to $30 \mathrm{MPa}$ confining pressure) are illustrated with crosses connected with yellow bars for comparison. The green dots display the results of the multiphase simulations (only performed for Carb-B and for $P$ waves). For details please refer to the text.

by void, and establishing a porosity-velocity trend. A more advanced technique using dry and wet imaging is suggested by Bhattad et al. (2014) using the difference imaging to approximate effective properties. However, the nanoindentation technique provides a measure for the distribution of effective elastic properties at the micrometre scale, and can thus potentially constrain the input parameters for the different phases identified during the segmentation. To be able to do so, nanoindentation needs to provide bulk and shear moduli from each of the measurements (load-displacement curves) and we need to obtain effective bulk and shear moduli values for each of the identified phases in the microtomography (pores, calcite grains, and the five micritic phases). However, if nanoindentation technique is a well-established technique in material sciences, which deals with homogeneous, purely elastic materials, this is, as of today, not the case for rocks, which are heterogeneous materials with both elastic and nonelastic behaviour (creep). Though nanoindentation tests pro- vide significant insights into elastic properties of heterogeneous rocks such as carbonates (Lebedev et al., 2014; Vialle and Lebedev, 2015) or shale (Ulm and Abousleiman, 2006; Abousleiman et al., 2007), there are still some points to be looked at before using the derived values of Young's (or shear and bulk) moduli in a quantitative way for DRP: the value of Poisson's ratio to be used, effect of surface roughness, local mechanical damage induced on the sample's surface by polishing techniques, etc. Nonetheless, the histograms of the indentation moduli of both samples show a broad distribution of moduli values ranging from very low values (a few GPa, where the indenter tip measures stiffness of an area mostly made of a pore) to values consistent with calcite. The existence of these intermediate values is consistent with the existence of micritic phases identified with X-ray tomography. However, we did not observe two peaks in the histogram for the nanoindentation results (Fig. 1) in contrast to the histograms of the scanned micro-XRCT images (Fig. 5). There- 

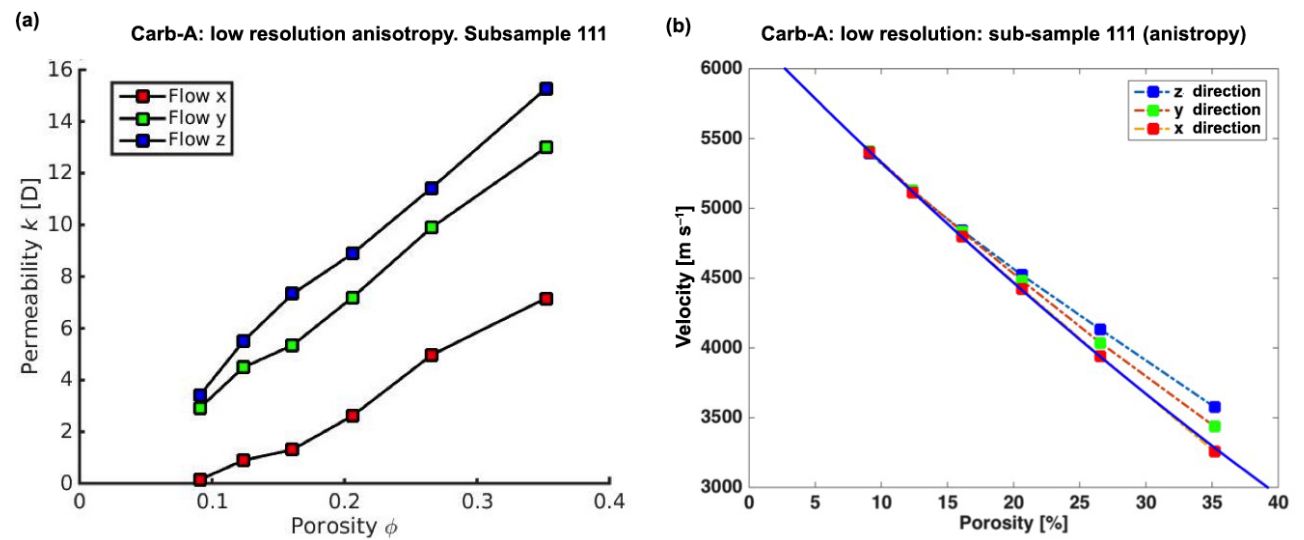

Figure 11. Left-hand side: intrinsic permeability for subvolume 111 of Carb-A low resolution. Permeability calculated from flow simulated in $x, y$, and $z$ directions. Right-hand side: $P$-wave velocities for all propagation directions of the same subsample. The solid line is the velocity trend given by Eq. (4). The moderate anisotropy visible in permeability is not present for the velocities.

fore the direct translation of moduli derived from nanoindentation also remains difficult. The resolution of nanoindentation used in this study allows for determining effective elastic properties at slightly bigger scales than those used here for the micro-XRCT.

Regnet et al. (2014) showed that there is a relationship between micrite microstructure and laboratory ultrasonic velocities on core samples, with samples with a higher content of tight micrite, exhibiting higher velocities, and samples with a higher content of microporous micrite, exhibiting lower velocities. Studied core samples were through a mixture of different types of micrite and the measured velocities represent effective properties at the core scale. This observation is reflected in the established porosity-velocity trends (Eqs. 4, 5, and 6): micritic phases with density closer to that of calcite (tight micrites) have higher velocities than micritic phases with lower density (Figs. 8 and 10), which are closer to that of pores (microporous micrites).

\subsection{Discussion of porosity: experiments vs. digital rock physics}

After the segmentation it is also possible to estimate the porosities of the samples. Based on the suggested workflow described in Sect. 3.2 there will be a lower and upper bound. For the lower bound we will treat only the high-confidence pores as pores; for the upper bound we count only highconfidence minerals as minerals.

We obtain a porosity range between 25 and $35 \%$ for the high-resolution data of Carb-A and a range between 7.5 and $31 \%$ for the low-resolution data. We observe that the mean value is in rough agreement with the experimentally determined porosity of $16.7 \%$ (see Table 1) only for the lowresolution case. Although also the experimental value comes with an error we conclude that the high-resolution data set for Carb-A is maybe not representative for the full sample used for the helium porosity in the laboratory. In case of CarbB the intervals range from 13 to 45 and 7 to $48 \%$ for the high-resolution and low-resolution case, respectively. Here the mean value is in both cases closer to the experimentally determined porosity of $29.4 \%$.

We conclude that the porosity values of carbonates using micro-XRCT data will only provide estimates with a relatively high uncertainty due to the significant amount of unresolved pore features in the images. An indication is the result of the mercury-intrusion experiments presented by Vialle et al. (2013): the pore throats of the micritic phase are mainly below the resolution of available micro-XRCT devices.

\subsection{Discussion of permeability: experiments vs. digital rock physics}

Permeability numerically estimated for Carb-B (Fig. 9, righthand side) presents an error of $97 \%$ on average with respect to the experimental value. In some cases the error is as low as $55 \%$. The numerical error in comparison with the experimental values is within the expected range for the numerical method at these porosities, cf. Table 1 in Osorno et al. (2015).

Experimental results for Carb-A sample are below the measurement error tolerance. This could imply a sample with no connected pores between the inlet and outlet defined for the experiment. The numerical estimation of the permeability for the Carb-A low-resolution sample (Fig. 9, left-hand side) is 4 orders of magnitude higher than the experimental measurement (on average 7.0 D). In the high-resolution case (Fig. 7, left-hand side) the numerical estimation is closer to experimental results (on average $0.1 \mathrm{D}$ ), but the porosity presents a large numerical error; therefore we do not take this domain as representative of the sample. However the numerically calculated permeability does not differ much from values found in the literature for porous rocks with similar porosity (cf. Andrä et al., 2013b). On the other hand it is ob- 
served that for a porosity below $25 \%$, permeability values of carbonates can span several orders of magnitude (e.g. Fig. 3 of Vialle et al., 2013). Therefore we suggest considering a statistically significant number of samples to characterize a formation and found that eight samples are sufficient for the numerical permeability calculations (see Figs. 7 and 9).

\subsection{Discussion of elasticity: experiments vs. digital rock physics}

There are two important observations. The two-phase trend (displayed with solid and dashed/dotted lines) seems to be an upper bound for velocities. This data-driven upper bound is much stricter than the bound given by Hashin-Shtrikman (see Fig. 8) and is now confirmed for several carbonates using several resolutions (this study and Saenger et al., 2014). Only for the low-resolution images we observe a slightly different trend for $P$ waves (Eq. 6 and Fig. 10).

The trend given by the envelope of the multiphase simulations (displayed by dashed/dotted lines in Fig. 10, righthand side) is not a strict lower bound, because the shape will strongly depend on the applied method to determine effective elastic properties for areas which are below the resolution limit of the used XRCT technique. The best choice to our knowledge is the two-phase trend discussed above, which can be regarded as a carbonate-data-driven effective medium approach. We suggest implementing here in the future also the findings of the nanoindentation experiments. However, we observe that the velocities obtained for the multiphase simulations are in a reasonable agreement with laboratory measurements. This is the case for a known porosity determined in complementary laboratory studies (see also Sect. 5.1). For carbonates the distribution of the micritic phases and their effective elastic behaviour is crucial to predict the effective wave speeds.

\subsection{Discussion of anisotropy: elasticity vs. permeability}

In general we do not observe any significant anisotropy for permeability and for velocities of the considered samples. However, a few samples are out of this general trend. One example is a subvolume of Carb-A (low-resolution case), for which we show the results for $P$-wave velocities and permeabilities in Fig. 11. Interestingly, the moderate anisotropy for the permeability is not present for the velocity.

\section{Summary}

With the current imaging techniques it remains difficult to resolve microstructures (on submicrometre scale) and image a representative volume at the same time, which is essential to understand the effective material properties of rocks. For this purpose the exact determination of the porosity of the rock samples is the most relevant parameter. To overcome this problem, we have conducted a specific multiphase seg- mentation technique and a careful calibration of DRP estimates with laboratory data. Especially for carbonate samples it is difficult to exactly estimate the porosity from raw CT data, because the micritic phases remain unresolved with an unknown porosity. Therefore, we use our presented numerical results in an inverse way. We suggest using the porosity determination from the laboratory and go back to our lowresolution trends given in Figs. 9 and 10. With a given porosity we can now estimate the permeability and the effective wave velocities.

In case of the studied samples Carb-A and Carb-B, we can predict $P$ and $S$-wave velocities with a good agreement to laboratory results. The presented two-phase trend (Eqs. 4, 5, and 6) is found to be an upper bound for a wide range of scales and can also be used as an effective medium approach to the micritic phases. The predicted permeability values are only in good agreement for Carb-B. Most probably the lowresolution image of Carb-A is not representative for the sample used in the laboratory.

However, for the used carbonate rock samples anisotropy seems insignificant for elastic as well as for hydraulic properties.

In general, the resolution of the XRCT is the limiting factor for the application of DRP for carbonate rock. The micritic phases remain unresolved even for the highest resolutions available. Therefore, the effective elastic properties have to be approximated. Our suggestion is to use the trend of the two-phase simulations. The implemented workflow in this paper can be applied in general for numerical estimates of mechanical and transport properties of carbonates. Because of the known strong heterogeneity of carbonates we suggest using a statistically significant amount of digital images to characterize a formation.

Acknowledgements. Erik H. Saenger would like to thank ExxonMobil for the support of some ideas presented in this study. This work was partially funded by Curtin Reservoir Geophysical Consortium (CRGC). The authors thank the National Geosequestration Laboratory (NGL) of Australia for providing access to the X-ray microscope Versa XRM 500 (Zeiss - XRadia) and to the Nanoindentation system (Fisher-Crips Laboratories Pty.Ltd.). Funding for this facility was provided by the Australian Federal Government.

Edited by: M. Halisch

Reviewed by: O. Lopez and one anonymous referee

\section{References}

Abousleiman, Y. N., Tran, M. H., Hoang, S., Bobko, C. P., Ortega, A., and Ulm, F. J.: Geomechanics field and laboratory characterization of the Woodford Shale: The next gas play, SPE Annual Technical Conference and Exhibition, 2007.

Andrä, H., Combaret, N., Dvorkin, J., Glatt, E., Han, J., Kabel, M., Keehm, Y., Krzikalla, F., Lee, M., Madonna, C., Marsh, M., 
Mukerji, T., Saenger, E. H., Sain, R., Saxena, N., Ricker, S., Wiegmann, A., and Zhan, X.: Digital rock physics benchmarksPart I: Imaging and segmentation, Comput. Geosci., 50, 25-32, 2013a.

Andrä, H., Combaret, N., Dvorkin, J., Glatt, E., Han, J., Kabel, M., Keehm, Y., Krzikalla, F., Lee, M., Madonna, C., Marsh, M., Mukerji, T., Saenger, E. H., Sain, R., Saxena, N., Ricker, S., Wiegmann, A., and Zhan, X.: Digital rock physics benchmarksPart II: Computing effective properties, Comput. Geosci., 50, 3343, 2013b.

Arns, C. H., Knackstedt, M. A., Pinczewskiz, M. V., and Garboczi, E. J.: Computation of linear elastic properties from microtomographic images: Methodology and agreement between theory and experiment, Geophysics, 67, 1396-1405, doi:10.1190/1.1512785, 2002.

Bhattad, P., Young, B., Berg, C. F., Rustad, A. B., and Lopez, O.: Xray micro-ct assisted drainage rock typing for characterization of flow behaviour of laminated sandstone reservoirs: International Symposium of the Society of Core Analysts, Avignon, France, SCA2014-024, 2014.

Blunt, M. J., Bijeljic, B., Dong, H., Gharbi, O., Iglauer, S., Mostaghimi, P., Paluszny, A., and Pentland, C.: Pore-scale imaging and modelling, Adv. Water Resour., 51, 197-216, doi:10.1016/j.advwatres.2012.03.003, 2013.

Cantrell, D. L. and Hagerty, R. M.: Microporosity in Arab Formation Carbonates, Saudi Arabia, GeoArabia, 4, 129-154, 1999.

Cnudde, V. and Boone, M. N.: High-resolution X-ray computed tomography in geosciences: A review of the current technology and applications, Earth-Sci. Rev., 123, 1-17, doi:10.1016/j.earscirev.2013.04.003, 2013.

Costanza-Robinson, M. S., Estabrook, B. D., and Fouhey, D. F.: Representative elementary volume estimation for porosity, moisture saturation, and air-water interfacial areas in unsaturated porous media: Data quality implications, Water Resour. Res., 47, W07513, doi:10.1029/2010WR009655, 2011.

Cremonini, G., Elmi, C., and Selli, R.: Note illustrative della carta geologica d'Italia. Foglio 156 "S. Marco in Lamis", Servizio Geologico d'Italia, 1971.

Derzhi, N., Dvorkin, J., Diaz, E.,Baldwin, C., Fang, Q., Sulayman, A., Soroka, W. L., Clark, A., Al Dayyni, T., and Kalam, Z: Comparison of traditional and digital rock physics techniques to determine the elastic core parameters in Cretaceous formations, Abu Dhabi, Abu Dhabi International Petroleum Exhibition \& Conference, Abu Dhabi, UAE, SPE-138586-PP, 2010.

Deville de Periere, M., Durlet, C., Vennin, E., Lambert, L., Bourillot, R., Caline, B., and Poli, E.: Morphometry of micrite particles in cretaceous microporous limestones of the Middle East: Influence on reservoir properties, Mar. Petrol. Geol., 28, 1727-1750, doi:10.1016/j.marpetgeo.2011.05.002, 2011.

Fischer-Cripps, A. C.: Nanoindentation, 2nd Edn., Springer-Verlag, ISBN 0387220453, 2004.

Fusseis, F., Xiao, X., Schrank, C., and De Carlo, F.: A brief guide to synchrotron radiation-based microtomography in (structural) geology and rock mechanics, J. Struct. Geol., 65, 1-16, doi:10.1016/j.jsg.2014.02.005, 2014.

Hill, R.: Elastic properties of reinforced solids: some theoretical principles, J. Mech. Phys. Solids, 11, 357-372, 1963.
Jouini, M. S., Vega, S., and Al-Ratrout, A.: Numerical estimation of carbonate rock properties using multiscale images, Geophys. Prospect., 63, 405-421 doi:10.1111/1365-2478.12156, 2015.

Klein, C. A. and Cardinale, C. F.: Young's modulus and Poisson's ratio of CVD diamond, Diam. Relat. Mater., 2, 918-923, 1992.

Lambert, L., Durlet, C., Loreau, J. P., and Marnier, G.: Burial dissolution of micrite in Middle East carbonate reservoirs (JurassicCretaceous): Keys for recognition and timing, Mar. Petrol. Geol., 23, 79-92, doi:10.1016/j.marpetgeo.2005.04.003, 2006.

Lebedev, M., Wilson, M. E. J., and Mikhaltsevitch, V.: An experimental study of solid matrix weakening in water-saturated Savonnières limestone, Geophys. Prospect., 62, 1253-1265, doi:10.1111/1365-2478.12168, 2014.

Lopez, O., Mock, A., Øren, P. E., Long, H., Kalam, Z., Vahrenkamp, V., Gibrata, M., Seraj, S., Chacko, S., Al Hammadi, M., Al Hosni, H., Sahn, H., and Vizamora, A.: Validation of fundamental carbonate reservoir core properties using digital rock physics, International Symposium of the Society of Core Analysts held in Aberdeen, Scotland, UK, SCA2012-19, 2012.

Madonna, C., Quintal, B., Frehner, M., Almqvist, B. S. G., Tisato, N., Pistone, M., Marone, F., and Saenger, E. H.: Synchrotronbased X-ray tomographic microscopy for rock physics investigations, Geophysics, 1, D53-D64, doi:10.1190/GEO2012-0113.1, 2013.

Martinis, B. and Pavan, G.: Note illustrative della carta geologica d'Italia, Foglio 157 "Monte S. Angelo": Servizio Geologico d'Italia, Roma, 1967.

Mavko, G., Mukerji, T., and Dvorkin, J.: The rock physics handbook, Cambridge University Press, 2009.

Moshier, S. O.: Microporosity in micritic limestones: A review, Sediment. Geol., 63, 191-213, doi:10.1016/00370738(89)90132-2, 1989.

Osorno, M., Uribe, D., Ruiz, O. E., and Steeb, H.: Finite Difference Calculations of Permeability in Large Domains in a Wide Porosity Range, Arch. Appl. Mech., 85, 1043-1054, doi:10.1007/s00419-015-1025-4, 2015.

Regnet, J. P., Robion, P., David, C., Fortin, J., Brigaud, B.,and Yven, B.: Acoustic and reservoir properties of microporous carbonate rocks: Implication of micrite particle size and morphology, J. Geophys. Res.-Sol. Ea., 120, 790-811, doi:10.1002/2014JB011313, 2014.

Ringstad, C., Westphal, E., Mock, M., Al Hammadi, M., Al Ratrout, A., and Kalam, Z.: Elastic Properties of Carbonate Reservoir Rocks Using Digital Rock Physics: 75th EAGE Conference \& Exhibition, London, 10-13 June 2013.

Saenger, E. H., Gold, N., and Shapiro, S. A.: Modeling the propagation of elastic waves using a modified finite-difference grid, Wave Motion, 31, 77-92, doi:10.1016/S0165-2125(99)00023-2, 2000.

Saenger, E. H., Krüger, O. S., and Shapiro, S. A.: Numerical considerations of fluid effects on wave propagation: Influence of the tortuosity, Geophys. Res. Lett., 31, L21613, doi:10.1029/2004GL020970, 2004.

Saenger, E. H., Enzmann, F., Keehm, Y., and Steeb, H.: Digital rock physics: Effect of fluid viscosity on effective elastic properties, J. Appl. Geophys., 74, 236-241, doi:10.1016/j.jappgeo.2011.06.001, 2011. 
Saenger, E. H., Madonna, C., Osorno, M., Uribe, D., and Steeb, H.: Digital Carbonate Rock Physics, SEG Annual Meeting, Denver, Colorado, 2014.

Saenger, E. H., Lebedev, M., Uribe, D., Osorno, M., Vialle, S., Duda, M., Inglauer, S., and Steeb, H.: Analysis of high resolution X-ray CT images of Bentheim sandstone under elevated confining pressure, Geophys. Prospect., 64, 848-859, doi:10.1111/1365-2478.12400, 2016.

Scotellaro, C., Vanorio, T., and Mavko, G.: The effect of mineral composition and pressure on carbonate rocks, 77th Annual International Meeting, SEG, Expanded Abstracts, 1684-1689, 2004.

Ulm, F.-J. and Abousleiman, Y.: The nanogranular nature of shale: Acta Geotechnica, 1, 77-88, doi:10.1007/s11440-006-0009-5, 2006.
Vialle, S. and Lebedev, M.: Heterogeneities in the elastic properties of microporous carbonate rocks at the microscale from nanoindentation tests, SEG Technical Program Expanded Abstracts, 3279-3284, doi:10.1190/segam2015-5885016.1, 2015.

Vialle, S., Dvorkin, J., and Mavko, G.: Implications of pore microgeometry heterogeneity for the movement and chemical reactivity of $\mathrm{CO}_{2}$ in carbonates, Geophysics, 78, L69-L86, 2013.

Wang, Y., Yang, Y., Xiao, T., Liu, K., Clennell, B., Zhang, G., and Wang, H.: Synchrotron-Based Data-Constrained Modeling Analysis of Microscopic Mineral Distributions in Limestone, International Journal of Geosciences, 4, 344-351, doi:10.4236/ijg.2013.42032, 2013. 